\title{
Uso Off-Label de BTX em Tratamento da Rosácea
}

\section{Off-Label Use of BTX in Rosacea Treatment}

DOI: $10.46919 / \operatorname{archv2n1-003~}$

Recebimento dos originais: 30/10/2020

Aceitação para publicação: 23/12/2020

\author{
Adriana Novaes Rodrigues \\ Doutora em Ciência pela Universidade de São Paulo \\ Instituição: Universidade de São Paulo \\ Endereço: Av. Dr. Arnaldo, 455 - Cerqueira César • CEP: 01246903 • São Paulo - SP - Brasil. \\ E-mail: a.novaes@live.com \\ Luisa Fernanda Guzmán \\ Pós graduação em Laser e pequenos procedimentos \\ Instituição: Faculdade de Ciências e Tecnologia \\ Endereço: Calle Josep Lansa 28-30, 2do - 4ta Vilanova I La Geltrú - Barcelona - Espanha \\ E-mail: luisafernandagb@ hotmail.com
}

\section{RESUMO}

Introdução: $\mathrm{O}$ eritema facial é uma característica clínica da rosácea e a injeção intradérmica de toxina botulínica tipo A (BoNT / A) tem sido usada off-label como uma forma de tratamento. Objetivo: o objetivo deste estudo é discutir esse tratamento, por meio do relato de um caso clínico. Métodos: Relato de caso, com discussão baseada em artigos indexados, sobre a melhora clínica do uso da toxina botulínica no tratamento da rosácea. Resultados: Melhora clínica considerável, a partir do segundo mês, após a aplicação. Conclusão: A aplicação da toxina botulínica mostrou-se eficaz no tratamento da rosácea

Palavras-chave: Avaliação clínica do eritema, Eritema, Rosácea, Toxina botulínica, Dermatologia.

\begin{abstract}
Background: Facial erythema is a clinical feature of rosacea and intradermal injection of botulinum toxin type A (BoNT / A) has been used off-label as a form of treatment. Objective: the objective of this study is to discuss this treatment, through the report of a clinical case. Methods: Case report, with discussion based on indexed articles, on the clinical improvement of the use of botulinum toxin in the treatment of rosacea. Results: Considerable clinical improvement, after the second month, after application. Conclusion: The application of botulinum toxin proved effective in the treatment of rosacea
\end{abstract}

Key words: Clinical evaluation of erythema, Erythema, Rosacea, Botulinum toxin, Dermatology.

\section{INTRODUÇÃO}

Rosácea é uma dermatose inflamatória comum, caracterizada por eritema persistente, telangiectasia, pápulas, pústulas e rubor facial ${ }^{1}$ e afeta principalmente a área facial central (ou seja, bochechas, nariz, queixo, testa e olhos $)^{2}$. Uma classificação padrão sistema, com base em características clínicas primárias, 
divide rosácea em 04 subgrupos: eritematotelangiectásica, papulopustular, fimatosa e ocular. ${ }^{2}$ A rosácea eritematotelangiectásica é caracterizada principalmente por rubor e eritema facial central persistente ${ }^{3 .}$

Para o tratamento são, atualmente, utilizados medicação oral, tópicos e terapia a laser são realizados rotineiramente, mas muitas vezes não conseguem aliviar o rubor facial ${ }^{4}$. Isto faz com que esta patologia afete consideravelmente a qualidade de vida dos pacientes e a busca de novas tratamento tem sido pesquisados, a fim de inimizar os transtornos.

Uma opção foi a investigação do uso de BTX -A em rosácea, por Odo et al. (2011), ${ }^{5}$ através de um estudo piloto, que demonstrou uma eficácia considerável, após terceiro mês da aplicação. Outros estudos, como de Kim, et al (2019), constataram, em pesquisa randomizada, que os resultados esperados de melhora, aparecem depois da segunda semana ${ }^{6}$

Com os resultados promissores, o objetivo deste artigo foi realizar uma discussão sobre o tema: rosácea e o uso toxina botulínica, através do relato de um caso clínico.

\section{MÉTODO}

Este estudo foi realizado de acordo com requisitos regulatórios, revisado e aprovado por revisão institucional, para relato de caso, com discussão baseada em artigos indexados. A paciente estava diagnostica usando as diretrizes padrão do National Rosacea Society Expert Committee, por um dermatologista.

No primeiro momento foi utilizado anestésico tópico (creme EMLA; Astra, Westborough, MA), aplicado por 30 minutos antes do tratamento e em seguida, completamente removido. A dose de diluição foi determinada pela revisão de relatórios de casos anteriores e ensaios clínicos, considerando tanto eficácia quanto segurança $^{7,8,9}$.

No segundo momento, após a remoção do creme anestésico tópico, foi tratado com BTX/A (Dysport; Ipsen Biopharm Ltd.) ${ }^{10}$ a uma diluição de 1 frasco: $7 \mathrm{~mL}$ soro salínico gelado (ou seja, um frasco de $500 \mathrm{U}$ de toxina foi reconstituído com $7 \mathrm{~mL}$ de soro salínico estéril, sem conservantes). Foi usado uma técnica de injeção intradérmica com uma seringa de $1 \mathrm{ml}$ e uma agulha $31-\mathrm{G}^{11}$

A aplicação de 30 UI, no total, em pontos pré determinados na face (terço médio - região malar), baseou-se em outras pesquisas ${ }^{12}$.

A paciente foi acompanhada por um período de 08 meses, após a aplicação e a documentação da evolução do quadro foi realizada através de fotografias. Apesar de poder ocorrer distorções pelo reflexo da luz, cor e profundidade, ainda clinicamente, é a melhor forma de registrar os resultados ${ }^{13}$, sendo assim apresentamos de forma visual os resultados obtidos. 


\section{DESCRIÇÃO DO CASO CLÍNICO}

Mulher, 53 anos, com quadro de rosácea diagnosticado há mais de 10 anos. Relatou piora importante do quadro dermatológico após o início da menopausa.

Em uso de levotiroxina $50 \mathrm{mg} / \mathrm{dia}$, há 20 anos e faz restrição alimentar dos alimentos em que ela considera de agravo a doença, como café e chá. Não tabagista e não etilista.

Realizou diversos tratamentos com medicações orais, tópicas, laser entre outros, sem melhora clínica. Sente-se constrangida com o eritema facial, deixando de sair para eventos públicos durante as crises de agravamento.

Exames laboratoriais dentro do padrão de normalidade.

Estava sem ser submetida a nenhum procedimento estético ou ter recebido tratamento com BTX no rosto há 6 meses.

\section{RESULTADOS}

O índice de eritema foi diminuído ao longo do período de estudo e os resultados mostraram melhora considerável, em dois meses após a aplicação (Foto 1 e Foto 2).

Mesmo com uso de anestésico tópico, a paciente relatou desconforto a aplicação. Na revisão, ela se expressou verbalmente sobre sua melhora em relação a qualidade de vida.

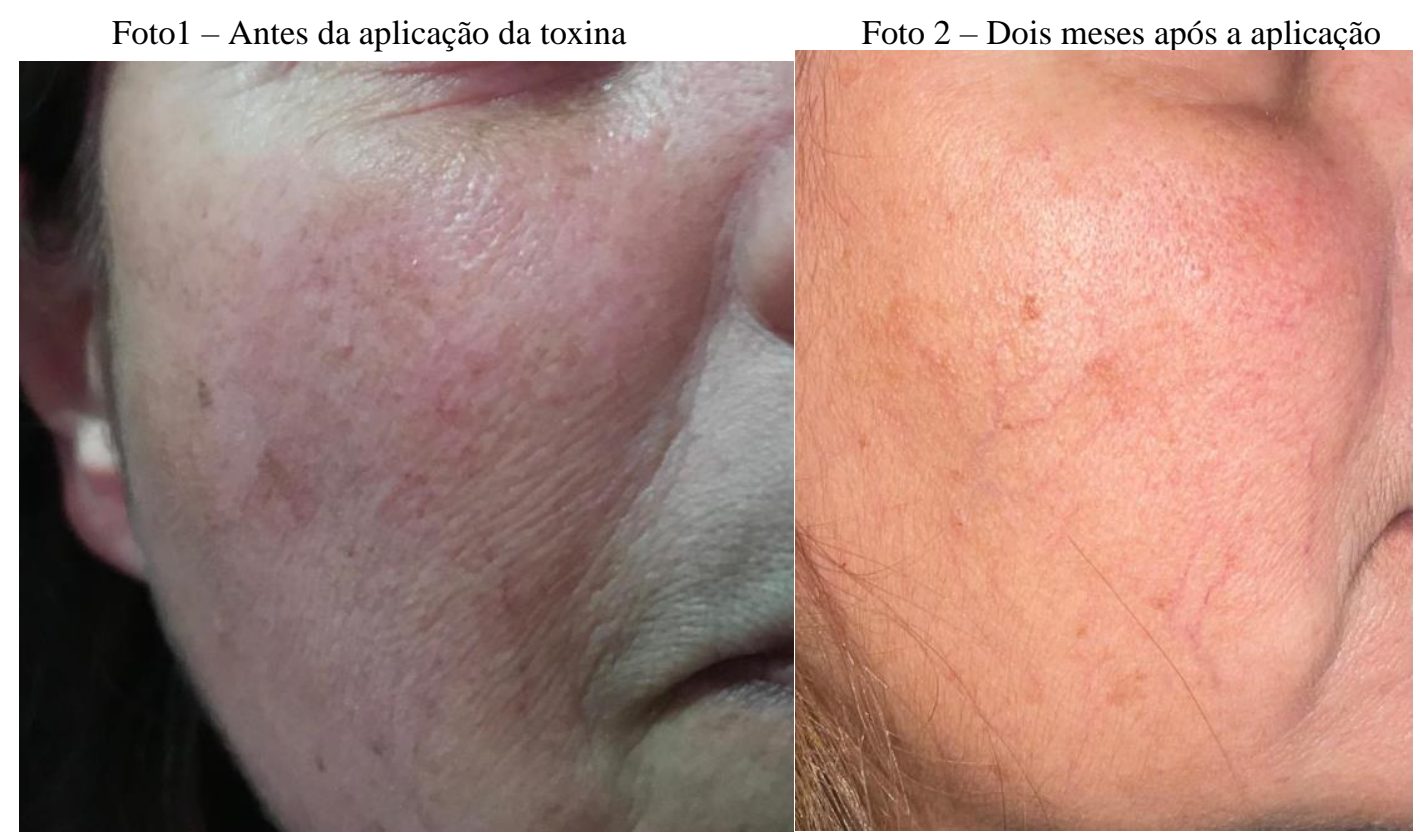




\section{DISCUSSÃO}

O eritema facial é uma característica clínica da rosácea e frequentemente causa sofrimento social e psicológico. A melhora clínica observada em dois meses após a aplicação, foi também observada por Bloom et al (2015) ${ }^{12}$ e Kim, et al (2019) ${ }^{6}$.

O uso off-label de BoNT / A em uma variedade de procedimentos cosméticos se tornaram mais populares nos últimos anos ${ }^{14}$

Desde a aprovação da utilização da toxina botulínica pela primeira vez em 2002 pelo FDA, muitos estudos para várias indicações de BTX, tem sido realizado. A eficácia da aplicação intradérmica das injeções de BTX para o tratamento de eritema facial é uma destas indicações. O primeiro relato da eficácia na melhora clínica do uso de BTX em rosácea, foi em 2002, por Yuraitis e Jacob ${ }^{15}$ Desde então, outros trabalhos relataram o efeito sobre a rosácea e ao eritema facial ${ }^{16}$., através de injeções intradérmicas de BTX. Outro efeito relatado é a diminuição da secreção de sebo ${ }^{15,17}$.

A toxina botulínica (BoNT) é uma neurotoxina produzida pela bactéria Clostridium botulinum. BoNT- e apresenta 07, sorotipos distintos $(\mathrm{A} \mathrm{a} \mathrm{G})^{18}$. Comercialmente a mais utilizada é a substância ativa é a holotoxina BoNT / A de $150 \mathrm{kDa}$. Esta holotoxina é sintetizada como uma única cadeia polipeptídica cortada pós-tradução para produzir os segmentos de cadeia pesada de $100 \mathrm{kDa}(\mathrm{HC})$ e cadeia leve de 50 $\mathrm{kDa}$ (LC), covalentemente ligados por uma ponte dissulfeto. Durante a síntese, a holotoxina forma complexos multiproteicos com a proteína não hemaglutinina não tóxica e os componentes hemaglutinina HA-70, HA-33 e HA-17 para gerar complexo de tamanho molecular diferente, o maior dos quais é o 900 kDa 19 S LL-PTC (complexo de toxina progenitora) ${ }^{19}$.

Em relação a eficácia e a potência, vários estudos relataram eficácia clínica inferior ou duração de ação mais curta para IBoNT em comparação com OBoNT. Da mesma forma, estudos não clínicos comparando a potência ou eficácia de OBoNT e IBoNT discordaram sobre sua equivalência de dose ${ }^{20}$.

O mecanismo de ação das neurotoxinas botulínicas é através da interrupção da fusão das vesículas sinápticas e da membrana plasmática em terminais nervosos colinérgicos periféricos clivando SNAREs (receptores de proteína de fixação de fator sensível a N-etilmaleimida solúvel) e prevenir a liberação de neurotransmissores de acetilcolina ( $\mathrm{ACh}$ ) nas junções neuromusculares. Este mecanismo de ação tornou a BoNT um agente terapêutico altamente adequado para distúrbios musculares relacionados à espasticidade 21.

No entanto, evidências recentes mostram o efeito de BoNTs em diferentes tipos de células humanas, tanto neuronais quanto não neuronais. Estes últimos incluem queratinócitos epidérmicos, células-tronco mesenquimais de tecido adiposo subcutâneo, neutrófilos e macrófagos, fibroblastos dérmicos, mastócitos, glândulas sebáceas e células endoteliais vasculares. Assim, várias aplicações clínicas estão surgindo na dermatologia 22 
A técnica de injeção intradérmica ou subdérmica, também conhecida como " micro-dosagem ",envolve o uso de uma concentração mais baixa de BoNT / A do que a formulação tradicional, para técnica de injeção intramuscular, sendo injetada em várias alíquotas menores nas áreas definidas para cada estudo 23. Esta técnica é a que mais tem sido utilizada de forma of label, nas pesquisas com patologias dermatológicas.

Em relação ao efeito sobre a melhora do eritema facial na rosácea, um possível mecanismo de ação é o bloqueio potente da acetilcolina através da liberação dos nervos periféricos autonômicos do sistema vasodilatador cutâneo, além de inibir a liberação de mediadores inflamatórios, como a substância P e calcitonina peptídeo relacionado ao gene (CGRP) ${ }^{24}$. com redução e o controle da inflamação cutânea local, podendo permitir que o eritema possa desaparecer ${ }^{25}$.

O desconforto relatado pela paciente não inviabiliza o procedimento, pois é uma das mais comuns queixas durante o tratamento com toxina botulínica ${ }^{26}$.

Em relação ao registro fotográfico e com base em um conjunto de fotografias de referência, a criação de um conjunto padrão de fotografias de referência e uma escala CEA de acompanhamento para uso em estudos, deveria ser criada, para melhor retratar a evolução clínica dos tratamentos ${ }^{27}$.

Apesar da rosácea não ter uma perspectiva de cura, uma alternativa terapêutica é a utilização da BTXA. Com possibilidades de melhora por longos períodos de remissão, há um impacto positivo na qualidade de vida destes pacientes.

\section{CONCLUSÃo}

A técnica de injeção intradérmica de BTX/A utilizada, nesta pesquisa, obteve uma melhora significativa, mesmo após o tempo estimado de ação pelo fabricante.

A melhora clínica possibilitou a melhora na qualidade de vida de vida da paciente.

A injeção intradérmica em micro dosagem, para rosácea demonstrou ser um uma opção viável de forma eficaz e segura para os pacientes.

Novos estudos, com aumento na significância de participantes devem ser desenvolvidos, para complementar os dados e firmar a terapêutica com BTX/A. 


\section{REFERENCIAS}

1. Crawford GH, Pelle MT, James WD. Rosacea, I: etiology, pathogenesis, and subtype classification. J Am Acad Dermatol 2004;51:327-41; quiz 342-4.

2. Odom R, Dahl M, Dover J, Draelos Z, Drake L, Macsai M, et al. Standard management options for rosacea, part 1: overview and broad spectrum of care. Cutis 2009;84:43-7

3. Tan, J., Liu, H., Leyden, J. J., \& Leoni, M. J. (2014). Reliability of Clinician Erythema Assessment grading scale. Journal of the American Academy of Dermatology, 71(4), 760-763. doi:10.1016/j.jaad.2014.05.044

4. Eshghi, G.; Khezrian, L.; Alirezaei, P. Botulinum toxin in treatment of facial flushing. Acta Med. Iran 2016, 54, 454-457.

5. Odo, M.E.; Odo, L.M.; Farias, R.V.; Primavera, R.A.; Leao, L.; Cuce, L.C.; Juliano, Y. Botulinum toxin for the treatment of menopausal hot flushes: A pilot study. Dermatol. Surg. 2011, 37, 1579-1583.

6. Kim MJ, Kim JH, Cheon HI, Hur MS, Han SH, Lee YW, Choe YB, Ahn KJ. Assessment of Skin Physiology Change and Safety After Intradermal Injections With Botulinum Toxin: A

Randomized, Double-Blind, Placebo-Controlled, Split-Face Pilot Study in Rosacea Patients With Facial Erythema. Dermatol Surg. 2019 Sep;45(9):1155-1162. doi: 10.1097/DSS.0000000000001819. PMID: 30730346.

7. Rose AE, Goldberg DJ. Safety and efficacy of intradermal injection of botulinum toxin for the treatment of oily skin. Dermatol Surg 2013;39:443-8.

8. Geddoa E, Matar HE, Paes TR. The use of botulinum toxin-A in the management of neck and anterior chest wall flushing: pilot study. Int J Dermatol 2013;52:1547-50.

9. Zhu J, Ji X, Xu Y, Liu J, et al. The efficacy of intradermal injection of type A botulinum toxin for facial rejuvenation. Dermatol Ther 2017;(1):1-4. doi: 10.1111/dth.12433.

10. US Food and Drug Administration. Dysport Highlights of prescribing information. https:// www.accessdata.fda.gov/drugsatfda_docs/label/ 2016/125274s107lbl.pdf. Accessed 18 Nov 2020]

11. Wanitphakdeedecha R, Ungaksornpairote C, Kaewkes A, et al. The comparison between intradermal injection of AbobotulinumtoxinA and normal saline for face-lifting: a split-face randomized controlled trial. J Cosmet Dermatol. 2016;15(4): 452-7

12. Bloom, B.S.; Payongayong, L.; Mourin, A.; Goldberg, D.J. Impact of intradermal abobotulinumtoxin A on facial erythema of rosacea. Dermatol. Surg. 2015, 41 (Suppl. 1), S9-S

13. Wanitphakdeedecha, R., Yan, C., Apinuntham, C., Rojanavanich, V., Cembrano, K. A. G., Eimpunth, S., \& Manuskiatti, W. (2020). Intradermal Micro-Dosing of AbobotulinumtoxinA for FaceLifting: How Long Does It Last? Dermatology and Therapy. doi:10.1007/s13555-020-00414-7

14. Campanati A, Martina E, Giuliodori K, Consales V, Boyr I, Offidani A. Botulinum toxin off-label use in dermatology: a review. Skin Appendage Disord. 2017;3(1):39-56 
15. Yuraitis M, Jacob CI. Botulinum toxin for the treatment of facial flushing. Dermatol Surg 2004;30:102-4..

16. Park KY, Hyun MY, Jeong SY, Kim BJ, et al. Botulinum toxin for the treatment of refractory erythema and flushing of rosacea. Dermatology 2015;230:299-301.

17. Rose AE, Goldberg DJ. Safety and efficacy of intradermal injection of botulinum toxin for the treatment of oily skin. Dermatol Surg 2013;39:443-8.

18. Franciosa G, Pourshaban M, De Luca A, Buccino A, et al. Identification of type A, B, E, and F botulinum neurotoxin genes and of botulinum neurotoxigenic clostridia by denaturing high-performance liquid chromatography. Appl Environ Microbiol 2004;70:4170-6.

19. Lam KH, Jin R. Architecture of the botulinum neurotoxin complex: a molecular machine for protection and delivery. Curr Opin Struct Biol 2015;31:89-95.

20. Wilson AJ, Chang B, Taglienti AJ, Chin BC, et al. A quantitative analysis of OnabotulinumtoxinA, AbobotulinumtoxinA, and IncobotulinumtoxinA: a randomized, double-blind, prospective clinical trial of comparative dynamic strain reduction. Plast Reconstr Surg 2016;137:1424-33

21. Guida S, Farnetani F, Nistico SP, Mariarosaria CG, et al. New trends in botulinum toxin use in dermatology. Dermatol Pract Concept 2018;8:277-82.

22. Binz T, Sikorra S, Mahrhold S. Clostridial neurotoxins: mechanism of SNARE cleavage and outlook on potential substrate specificity reengineering. Toxins (Basel) 2010;2:665-82

23. Wu WT. Microbotox of the lower face and neck: evolution of a personal technique and its clinical effects. Plast Reconstr Surg. 2015;136(5 Suppl): 92S-100S.

24. A Carmichael, M.M.; Dostrovsky, J.O.; Charlton, M.P. Peptide-mediated transdermal delivery of botulinum neurotoxin type A reduces neurogenic inflammation in the skin. Pain 2010, 149, 316-324.

25. Tugnoli V, Marchese Ragona R, Eleopra R, et al. The role of gustatory flushing in Frey's syndrome and its treatment with botulinum toxin type A. Clin Auton Res. 2002;12(3):174-178.

26. Rodrigues, A. N..; Franco, M. F. M. N. Revisão das Complicações da Utilização da Toxina Botulínica Full Face. Archives of Health, v. 1, n. 6, p. 577-583, 22 Dec. 2020. doi:10.46919/archv1n6-018

27. Tan, J., Liu, H., Leyden, J. J., \& Leoni, M. J. (2014). Reliability of Clinician Erythema Assessment grading scale. Journal of the American Academy of Dermatology, 71(4), 760-763. doi:10.1016/j.jaad.2014.05.044 